\title{
Lipschitz shadowing implies structural stability
}

\author{
Sergei Yu. Pilyugin *and Sergey Tikhomirov ${ }^{\dagger}$
}

\begin{abstract}
We show that Lipschitz shadowing property of a diffeomorphism is equivalent to structural stability. As a corollary, we show that an expansive diffeomorphism having Lipschitz shadowing property is Anosov.
\end{abstract}

Keywords: Lipschitz shadowing, hyperbolicity, structural stability

\section{Introduction}

The theory of shadowing of approximate trajectories (pseudotrajectories) of dynamical systems is now a well developed part of the global theory of dynamical systems (see, for example, the monographs [1, 2]).

This theory is closely related to the classical theory of structural stability. It is well known that a diffeomorphism has shadowing property in a neighborhood of a hyberbolic set [3, 4] and a structurally stable diffeomorphism has shadowing property on the whole manifold [5, 6, 7]. Analyzing the proofs of the first shadowing results by Anosov [3] and Bowen [4], it is easy to see that, in a neighborhood of a hyperbolic set, the shadowing property is Lipschitz (and the same holds in the case of a structurally stable diffeomorphism, see [1]).

${ }^{* 1}$ Faculty of Mathematics and Mechanics, St. Petersburg State University, University av. 28, 198504, St. Petersburg, Russia..sp@sp1196.spb.edu

${ }^{\dagger}$ Department of Mathematics, National Taiwan University, No. 1, Section 4, Roosevelt Road, Taipei 106, Taiwan. sergey.tikhomirov@gmail.com 
At the same time, it is easy to give an example of a diffeomorphism that is not structurally stable but has shadowing property (see [8], for example).

Thus, structural stability is not equivalent to shadowing.

One of possible approaches in the study of relations between shadowing and structural stability is the passage to $C^{1}$-interiors. At present, it is known that the $C^{1}$-interior of the set of diffeomorphisms having shadowing property coincides with the set of structurally stable diffeomorphisms [9]. Later, a similar result was obtained for orbital shadowing property (see [10] for details).

Here, we are interested in the study of the above-mentioned relations without the passage to $C^{1}$-interiors. Let us mention in this context that Abdenur and Diaz conjectured that a $C^{1}$-generic diffeomorphism with the shadowing property is structurally stable; they have proved this conjecture for so-called tame diffeomorphisms [11]. Recently, the first author has proved that the so-called variational shadowing is equivalent to structural stability [8].

In this short note, we show that Lipschitz shadowing property is equivalent to structural stability.

As a corollary, we show that an expansive diffeomorphism having Lipschitz shadowing property is Anosov. Let us mention that Ombach [12] and Walters [13] showed that a diffeomorphism $f$ is Anosov if and only if $f$ has shadowing property and is strongly expansive (which means that all the diffeomorphisms in a $C^{1}$-small neighborhood of $f$ are expansive with the same expansivity constant).

In addition, let us mention the recent paper [14, where it is shown that Lipschitz periodic shadowing is equivalent to $\Omega$-stability.

\section{Main result}

Let us pass to exact definitions and statements.

Let $f$ be a diffeomorphism of class $C^{1}$ of an $m$-dimensional closed smooth manifold $M$ with Riemannian metric dist. Let $D f(x)$ be the differential of $f$ at a point $x$. For a point $p \in M$, we denote $p_{k}=f^{k}(p), k \in \mathbb{Z}$.

Denote by $T_{x} M$ the tangent space of $M$ at a point $x$; let $|v|, v \in T_{x} M$, be the norm of $v$ generated by the metric dist.

We say that $f$ has shadowing property if for any $\epsilon>0$ there exists $d>0$ with the following property: for any sequence of points $X=\left\{x_{k} \in M\right\}$ such 
that

$$
\operatorname{dist}\left(x_{k+1}, f\left(x_{k}\right)\right)<d, \quad k \in \mathbb{Z},
$$

there exists a point $p \in M$ such that

$$
\operatorname{dist}\left(x_{k}, p_{k}\right)<\epsilon, \quad k \in \mathbb{Z}
$$

(if inequalities (11) hold, one says that the trajectory $\left\{p_{k}\right\}$-shadows the $d$ pseudotrajectory $X$ ).

We say that $f$ has Lipschitz shadowing property if there exist constants $\mathcal{L}, d_{0}>0$ with the following property: For any $d$-pseudotrajectory $X$ as above with $d \leq d_{0}$ there exists a point $p \in M$ such that

$$
\operatorname{dist}\left(x_{k}, p_{k}\right) \leq \mathcal{L} d, \quad k \in \mathbb{Z}
$$

The main result of this note is the following statement.

Theorem 1. The following two statements are equivalent:

(1) f has Lipschitz shadowing property;

(2) $f$ is structurally stable.

Remark 2. Let us recall that a diffeomorphism $f$ is called expansive if there exists a positive number $a$ (expansivity constant) such that if $x, y \in M$ and

$$
\operatorname{dist}\left(f^{k}(x), f^{k}(y)\right) \leq a
$$

for all $k \in \mathbb{Z}$, then $x=y$. The above theorem has the following corollary.

Corollary 3. The following two statements are equivalent:

(1) $f$ is expansive and has Lipschitz shadowing property;

(2) $f$ is Anosov.

Proof of the corollary. The implication $(2) \Rightarrow(1)$ is well known (see, for example, [1]). By our theorem, condition (1) of the corollary implies that $f$ is structurally stable, and it was shown by Mañe that an expansive structurally stable diffeomorphism is Anosov (see [15]). 
Now we pass to the proof of the main theorem.

The implication $(2) \Rightarrow(1)$ is well known (see, for example, [1]).

In the proof of the implication $(1) \Rightarrow(2)$, we use the following two known results (Propositions 4 and 5).

First we introduce some notation. For a point $p \in M$, define the following two subspaces of $T_{p} M$ :

$$
B^{+}(p)=\left\{v \in T_{p} M:\left|D f^{k}(p) v\right| \rightarrow 0, \quad k \rightarrow+\infty\right\}
$$

and

$$
B^{-}(p)=\left\{v \in T_{p} M:\left|D f^{k}(p) v\right| \rightarrow 0, \quad k \rightarrow-\infty\right\} .
$$

Proposition 4. [Mañé, [16]]. The diffeomorphism $f$ is structurally stable if and only if

$$
B^{+}(p)+B^{-}(p)=T_{p} M
$$

for any $p \in M$.

Consider a sequence of linear isomorphisms

$$
\mathcal{A}=\left\{A_{k}: \mathbb{R}^{m} \rightarrow \mathbb{R}^{m}, k \in \mathbb{Z}\right\}
$$

for which there exists a constant $N>0$ such that $\left\|A_{k}\right\|,\left\|A_{k}^{-1}\right\| \leq N$. Fix two indices $k, l \in \mathbb{Z}$ and denote

$$
\Phi(k, l)= \begin{cases}A_{k-1} \circ \cdots \circ A_{l}, & l<k \\ \mathrm{Id}, & l=k \\ A_{k}^{-1} \circ \cdots \circ A_{l-1}^{-1}, & l>k\end{cases}
$$

Set

$$
B^{+}(\mathcal{A})=\left\{v \in \mathbb{R}^{m}:|\Phi(k, 0) v| \rightarrow 0, \quad k \rightarrow+\infty\right\}
$$

and

$$
B^{-}(\mathcal{A})=\left\{v \in \mathbb{R}^{m}:|\Phi(k, 0) v| \rightarrow 0, \quad k \rightarrow-\infty\right\} .
$$

Proposition 5. [Pliss, [17]] The following two statements are equivalent:

(a) For any bounded sequence $\left\{w_{k} \in \mathbb{R}^{m}, k \in \mathbb{Z}\right\}$ there exists a bounded sequence $\left\{v_{k} \in \mathbb{R}^{m}, k \in \mathbb{Z}\right\}$ such that

$$
v_{k+1}=A_{k} v_{k}+w_{k}, \quad k \in \mathbb{Z}
$$


(b) the sequence $\mathcal{A}$ is hyperbolic on any of the rays $[0,+\infty)$ and $(-\infty, 0]$ (see the definition in [18]), and the spaces $B^{+}(\mathcal{A})$ and $B^{-}(\mathcal{A})$ are transverse.

Remark 6. In fact, Pliss considered in [17] not sequences of isomorphisms but homogeneous linear systems of differential equations; the relation between these two settings is discussed in [18].

Remark 7. Later, a statement analogous to Proposition [5 was proved by Palmer [19, 20]; Palmer also described Fredholm properties of the corresponding operator

$$
\left\{v_{k} \in \mathbb{R}^{m}: k \in \mathbb{Z}\right\} \mapsto\left\{v_{k}-A_{k-1} v_{k-1}\right\} .
$$

We fix a point $p \in M$ and consider the isomorphisms

$$
A_{k}=D f\left(p_{k}\right): T_{p_{k}} M \rightarrow T_{p_{k+1}} M .
$$

Note that the implication $(\mathrm{a}) \Rightarrow(\mathrm{b})$ of Proposition 5 is valid for the sequence $\left\{A_{k}\right\}$ with $\mathbb{R}^{m}$ replaced by $T_{p_{k}} M$.

It follows from Propositions 4 and 5 that our main theorem is a corollary of the following statement (indeed, we prove that Lipschitz shadowing property implies the validity of statement (a) of Proposition 5 for any trajectory $\left\{p_{k}\right\}$ of $f$, while the validity of statement (b) of this proposition implies the structural stability of $f$ by Proposition 4).

Lemma 8. If $f$ has the Lipschitz shadowing property with constants $\mathcal{L}, d_{0}$, then for any sequence $\left\{w_{k} \in T_{p_{k}} M, k \in \mathbb{Z}\right\}$ such that $\left|w_{k}\right|<1, k \in \mathbb{Z}$, there exists a sequence $\left\{v_{k} \in T_{p_{k}} M, k \in \mathbb{Z}\right\}$ such that

$$
\left|v_{k}\right| \leq 8 \mathcal{L}+1, \quad v_{k+1}=A_{k} v_{k}+w_{k}, \quad k \in \mathbb{Z} .
$$

To prove Lemma 8 , we first prove the following statement.

Lemma 9. Assume that $f$ has the Lipschitz shadowing property with constants $\mathcal{L}, d_{0}$. Fix a trajectory $\left\{p_{k}\right\}$ and a natural number $n$. For any sequence $\left\{w_{k} \in T_{p_{k}} M, k \in[-n, n]\right\}$ such that $\left|w_{k}\right|<1$ for $k \in[-n, n]$ and $w_{k}=0$ for $k \notin[-n, n]$ there exists a sequence $\left\{z_{k} \in T_{p_{k}} M, k \in \mathbb{Z}\right\}$ such that

$$
\left|z_{k}\right| \leq 8 \mathcal{L}+1, \quad k \in \mathbb{Z}
$$

and

$$
z_{k+1}=A_{k} z_{k}+w_{k}, \quad k \in[-n, n] .
$$


Proof. First we locally "linearize" the diffeomorphism $f$ in a neighborhood of the trajectory $\left\{p_{k}\right\}$.

Let exp be the standard exponential mapping on the tangent bundle of $M$ and let $\exp _{x}: T_{x} M \rightarrow M$ be the corresponding exponential mapping at a point $x$.

We introduce the mappings

$$
F_{k}=\exp _{p_{k+1}}^{-1} \circ f \circ \exp _{p_{k}}: T_{p_{k}} M \rightarrow T_{p_{k+1}} M .
$$

It follows from the standard properties of the exponential mapping that $D \exp _{x}(0)=\mathrm{Id}$; hence, $D F_{k}(0)=A_{k}$. Since $M$ is compact, for any $\mu>0$ we can find $\delta>0$ such that if $|v| \leq \delta$, then

$$
\left|F_{k}(v)-A_{k} v\right| \leq \mu|v|
$$

Denote by $B(r, x)$ the ball in $M$ of radius $r$ centered at a point $x$ and by $B_{T}(r, x)$ the ball in $T_{x} M$ of radius $r$ centered at the origin.

There exists $r>0$ such that, for any $x \in M$, $\exp _{x}$ is a diffeomorphism of $B_{T}(r, x)$ onto its image, and $\exp _{x}^{-1}$ is a diffeomorphism of $B(r, x)$ onto its image. In addition, we may assume that $r$ has the following property.

If $v, w \in B_{T}(r, x)$, then

$$
\frac{\operatorname{dist}\left(\exp _{x}(v), \exp _{x}(w)\right)}{|v-w|} \leq 2
$$

if $y, z \in B(r, x)$, then

$$
\frac{\left|\exp _{x}^{-1}(y)-\exp _{x}^{-1}(z)\right|}{\operatorname{dist}(y, z)} \leq 2 .
$$

Now we pass to construction of pseudotrajectories; every time, we take $d$ so small that the considered points of our pseudotrajectories, points of shadowing trajectories, their "lifts" to tangent spaces etc belong to the corresponding balls $B\left(r, p_{k}\right)$ and $B_{T}\left(r, p_{k}\right)$ (and we do not repeat this condition on the smallness of $d$ ).

Fix a sequence $w_{k}$ having the properties stated in Lemma 9. Consider the sequence $\left\{\Delta_{k} \in T_{p_{k}} M, k \in[-n, n+1]\right\}$ defined as follows:

$$
\left\{\begin{array}{l}
\Delta_{-n}=0 \\
\Delta_{k+1}=A_{k} \Delta_{k}+w_{k}, \quad k \in[-n, n] .
\end{array}\right.
$$


Let $Q=\max _{k \in[-n, n+1]}\left|\Delta_{k}\right|$.

Fix a small $d>0$ and construct a pseudotrajectory $\left\{\xi_{k}\right\}$ as follows:

$$
\begin{cases}\xi_{k}=\exp _{p_{k}}\left(d \Delta_{k}\right), & k \in[-n, n+1], \\ \xi_{l}=f^{l+n}\left(\xi_{-n}\right), & l \leq-n-1, \\ \xi_{l}=f^{l-n-1}\left(\xi_{n+1}\right), & l>n+1 .\end{cases}
$$

Note that definition (9) of the vectors $\Delta_{k}$ and condition (7) imply that if $d$ is small enough, then the following inequality holds:

$$
\operatorname{dist}\left(\xi_{k+1}, \exp _{p_{k+1}}\left(d A_{k} \Delta_{k}\right)\right)<2 d .
$$

Since

$$
f\left(\xi_{k}\right)=\exp _{p_{k+1}}\left(F_{k}\left(d \Delta_{k}\right)\right),
$$

condition (6) with $\mu<1$ implies that if $d$ is small enough, then

$$
\operatorname{dist}\left(\exp _{p_{k+1}}\left(d A_{k} \Delta_{k}\right), f\left(\xi_{k}\right)\right)<2 d .
$$

Hence,

$$
\operatorname{dist}\left(f\left(\xi_{k}\right), \xi_{k+1}\right) \leq 4 d .
$$

Let us note that the required smallness of $d$ is determined by the chosen trajectory $\left\{p_{k}\right\}$, the sequence $w_{k}$, and the number $n$.

The Lipschitz shadowing property of $f$ implies that if $d$ is small enough, then there exists an exact trajectory $\left\{y_{k}\right\}$ such that

$$
\operatorname{dist}\left(\xi_{k}, y_{k}\right) \leq 4 \mathcal{L} d, \quad k \in[-n, n+1] .
$$

Consider the finite sequence

$$
\left\{t_{k}=\frac{1}{d} \exp _{p_{k}}^{-1}\left(y_{k}\right), k \in[-n, n+1]\right\} .
$$

Inequalities (101) and (8) imply that

$$
\left|\Delta_{k}-t_{k}\right|<8 \mathcal{L} .
$$

Consider the finite sequence $\left\{b_{k} \in T_{p_{k}} M, k \in[-n, n+1]\right\}$ defined as follows:

$$
b_{-n}=t_{-n}, \quad b_{k+1}=A_{k} b_{k}, \quad k \in[-n, n] .
$$


Obviously, the following inequalities hold for $k \in[-n, n+1]$ :

$$
\operatorname{dist}\left(y_{k}, p_{k}\right) \leq \operatorname{dist}\left(y_{k}, \xi_{k}\right)+\operatorname{dist}\left(p_{k}, \xi_{k}\right) \leq 4 \mathcal{L} d+2 d\left|\Delta_{k}\right| \leq 2(Q+2 \mathcal{L}) d
$$

These inequalities and inequalities (8) imply that

$$
\left|t_{k}\right| \leq 4(Q+2 \mathcal{L})
$$

Take $\mu_{1}>0$ such that

$$
\left((N+1)^{2 n}+(N+1)^{2 n-1}+\cdots+1\right) \mu_{1}<1
$$

where $N=\sup \left\|A_{k}\right\|$.

Set

$$
\mu=\frac{\mu_{1}}{4(Q+2 \mathcal{L})}
$$

and consider $d$ so small that inequality (6) holds for $\delta=4(Q+2 L) d$.

The definition of the vectors $t_{k}$ implies that $d t_{k+1}=F_{k}\left(d t_{k}\right)$; since

$$
\left|d t_{k}\right| \leq 4 d(Q+2 \mathcal{L})
$$

by (13), we deduce from estimate (6) applied to $v=d t_{k}$ that

$$
\left|d t_{k+1}-d A_{k} t_{k}\right| \leq \mu d\left|t_{k}\right|
$$

Now we deduce from inequalities (13) that

$$
\left|t_{k+1}-A_{k} t_{k}\right| \leq 4 \mu(Q+2 \mathcal{L})=\mu_{1}, \quad k \in[-n, n] .
$$

Consider the sequence $c_{k}=t_{k}-b_{k}$. Note that $c_{-n}=0$ by (12). Estimates (15) imply that $\left|c_{k+1}-A_{k} c_{k}\right| \leq \mu_{1}$. Hence,

$$
\left|c_{k}\right| \leq\left((N+1)^{2 n}+(N+1)^{2 n-1}+\cdots+1\right) \mu_{1}<1, \quad k \in[-n, n] .
$$

Thus,

$$
\left|t_{k}-b_{k}\right|<1 \text {. }
$$

Consider the sequence $\left\{z_{k} \in T_{p_{k}} M, k \in \mathbb{Z}\right\}$ defined as follows:

$$
\begin{cases}z_{k}=\Delta_{k}-b_{k}, & k \in[-n, n+1], \\ z_{k}=0, & k \notin[-n, n+1] .\end{cases}
$$

Inequalities (11) and (16) imply estimate (44), while equalities (9) and (12) imply relations (5). Lemma 9 is proved. 
Proof of Lemma 8. Fix $n>0$ and consider the sequence

$$
w_{k}^{(n)}= \begin{cases}w_{k}, & k \in[-n, n] \\ 0, & |k|>n\end{cases}
$$

By Lemma 9, there exists a sequence $\left\{z_{k}^{(n)} \in T_{p_{k}} M, k \in \mathbb{Z}\right\}$ such that

$$
\left|z_{k}^{(n)}\right| \leq 8 \mathcal{L}+1, \quad k \in \mathbb{Z},
$$

and

$$
z_{k+1}^{(n)}=A_{k} z_{k}^{(n)}+w_{k}^{(n)}, \quad k \in[-n, n] .
$$

Passing to a subsequence of $\left\{z_{k}^{(n)}\right\}$, we can find a sequence $\left\{v_{k} \in T_{p_{k}} M, k \in\right.$ $\mathbb{Z}\}$ such that

$$
v_{k}=\lim _{n \rightarrow \infty} z_{k}^{(n)}, \quad k \in \mathbb{Z} .
$$

(Let us note that we do not assume uniform convergence.) Passing to the limit in estimates (17) and equalities (18) as $n \rightarrow \infty$, we get relations (3). Lemma 8 and our theorem are proved.

\section{Acknowledgement}

The research of the second author is supported by NSC (Taiwan) 98-2811M-002-061.

\section{References}

[1] S. Yu. Pilyugin, Shadowing in Dynamical Systems, Lecture Notes Math., vol. 1706, Springer, Berlin, 1999.

[2] K. Palmer, Shadowing in Dynamical Systems. Theory and Applications, Kluwer, Dordrecht, 2000.

[3] D. V. Anosov, On a class of invariant sets of smooth dynamical systems, Proc. 5th Int. Conf. on Nonlin. Oscill., 2, Kiev, 1970, 39-45.

[4] R. Bowen, Equilibrium States and the Ergodic Theory of Anosov Diffeomorphisms, Lecture Notes Math., vol. 470, Springer, Berlin, 1975. 
[5] C. Robinson, Stability theorems and hyperbolicity in dynamical systems, Rocky Mount. J. Math., 7, 1977, 425-437.

[6] A. Morimoto, The method of pseudo-orbit tracing and stability of $d y$ namical systems, Sem. Note 39, Tokyo Univ., 1979.

[7] K. Sawada, Extended $f$-orbits are approximated by orbits, Nagoya Math. J., 79, 1980, 33-45.

[8] S. Yu. Pilyugin, Variational shadowing (to appear).

[9] K. Sakai, Pseudo orbit tracing property and strong transversality of diffeomorphisms of closed manifolds, Osaka J. Math., 31, 1994, 373-386.

[10] S. Yu. Pilyugin, A. A. Rodionova, and K. Sakai, Orbital and weak shadowing properties, Discrete Contin. Dyn. Syst., 9, 2003, 287-308.

[11] F. Abdenur and L. J. Diaz, Pseudo-orbit shadowing in the $C^{1}$ topology, Discrete Contin. Dyn. Syst., 7, 2003, 223-245.

[12] J. Ombach, Shadowing, expansiveness, and hyperbolic homeomorphisms, J. Austral. Math. Soc, Ser. A, 61, 1996, 57-72.

[13] P. Walters, On the pseudo-orbit tracing property and its relationship to stability, in: The Structure of Attractors in Dynamical Systems, Lecture Notes Math., vol. 668, Springer, Berlin, 1978, 231-244.

[14] A. V. Osipov, S. Yu. Pilyugin, and S. B. Tikhomirov, Periodic shadowing and $\Omega$-stability (submitted to Regular and Chaotic Dynamics).

[15] R. Mañé, Expansive diffeomorphisms, in: Dynamical Systems - Warwick 1974, Lecture Notes Math., vol. 468, Springer, Berlin, 1975, 162-174.

[16] R. Mañé, Characterizations of AS diffeomorphisms, in: Geometry and Topology, Lecture Notes Math., vol. 597, Springer, Berlin, 1977, 389394.

[17] V. A. Pliss, Bounded solutions of nonhomogeneous linear systems of differential equations, Probl. Asympt. Theory Nonlin. Oscill., Kiev, 1977, 168-173. 
[18] S. Yu. Pilyugin, Generalizations of the notion of hyperbolicity, J. Difference Equat. Appl., 12, 2006, 271-282.

[19] K. J. Palmer, Exponential dichotomies and transversal homoclinic points, J. Differ. Equat., 55, 1984, 225-256.

[20] K. J. Palmer, Exponential dichotomies and Fredholm operators, Proc. Amer. Math. Soc., 104, 1988, 149-156. 